\title{
Inventory prediction and management in Nigeria using market basket analysis associative rule mining: memetic algorithm based approach
}

\author{
Arnold Adimabua Ojugo ${ }^{1}$, Andrew Okonji Eboka ${ }^{2}$ \\ ${ }^{1}$ Department of Mathematics/Computer Science, Federal University of Petroleum Resources Effurun, Nigeria \\ ${ }^{2}$ Department of Computer Science Education, Federal College of Education (Technical) Asaba, Nigeria
}

\begin{tabular}{l} 
Article Info \\
\hline Article history: \\
Received Jul 12, 2019 \\
Revised Nov 13, 2019 \\
Accepted Nov 28, 2019 \\
\hline Keywords: \\
Associative rule mining \\
Concept drift \\
Data mining \\
Market basket \\
Prediction analysis \\
Shelve placement \\
\hline
\end{tabular}

\begin{abstract}
A well-prepared abstract enables the reader to identify the basic content of a document quickly and accurately, to determine its relevance to their interests, and thus to decide whether to read the document in its entirety. The Abstract should be informative and completely self-explanatory, provide a clear statement of the problem, the proposed approach or solution, and point out major findings and conclusions. The Abstract should be 100 to 200 words in length. The abstract should be written in the past tense. Standard nomenclature should be used and abbreviations should be avoided. No literature should be cited. The keyword list provides the opportunity to add keywords, used by the indexing and abstracting services, in addition to those already present in the title. Judicious use of keywords may increase the ease with which interested parties can locate our article.
\end{abstract}

Copyright (c) 2019 Institute of Advanced Engineering and Science. All rights reserved.

\section{Corresponding Author:}

Arnold Adimabua Ojugo,

Department of Mathematics/Computer Science,

Federal University of Petroleum Resources Effurun,

P.M.B 1221, Effurun, Warri, Delta State, Nigeria.

Email: maryarnoldojugo@gmail.com

\section{INTRODUCTION}

The world over, businesses take stock and inventories of their daily production so as to account for goods and services rendered to their client in exchange for money. Inventories have been viewed by many as raw materials, work in progress or finished products that are stored to meet the supply demands of consumers $[1,2]$. If the amount of inventory is less than amount of actual need, the business may lose the opportunity to maximize sales. They may also lose potential clients, lose loyalties as well as lose anticipated maximum profits; While, if they stock too much of the inventory, it will increase the cost of maintenance and storage, and also consequently - reduce the profit margins [3, 4].

Inventory supply demand value chain and its management have rippled many businesses with a range of complications. Thus, the field has attracted the attention of many researchers and practitioners nowadays. [5] used moving average model for a company with fluctuating demand, proved that moving average is able to accommodate rapid changes in data; And quite suitable for companies with conditions of high variety of products and raw materials. This method is appropriate when used to predict long-term predictions. Thus, [5] explored other studies that employed exponential smoothing model [6] and the Box-Jenkins auto-regressive integrated moving average [7]. The inherent limitations of each method and others, accounted for the difficulty in applying the method to knowledge-base. However, [8] employed genetic algorithm to predict inventory stocks and proved that memetic algorithm offers many benefits such as its being a computational more efficient algorithm, more accurate and less time-consuming. In furtherance, [9] extended this work using deep learning and noted that there are inherent challenges in using genetic algorithm. That while, artificial neural networks 
(ANN) are suited for learning the underlying probabilities of feats of interest in market basket analysis - deep learning is best suited to predict the amount of product inventory needs due to other spatial data therein like prediction of concept evolution, concept drift amongst others. Thus, the rationale and mainstay focus for using memetic algorithm here is to advance the use of ANN in prediction of inventory as here proposed.

MBA is a data mining method, focusing on identification of products that are purchased at same time on each transaction [10]. Output of MBA is a set of rules that indicate the products that are purchased on the same time. This output will be used as input for the prediction of inventory. The rules generated by MBA are association rule(s) are of the form: If antecedent (A), then consequent (B). Each rule is equipped with a support level that indicates the number of transactions containing $\mathrm{A}$ and $\mathrm{B}$ and confidence level that is a measure of accuracy which is the rule of association rules. $[11,12]$ each rule is also equipped with an expected condition and a lift so that for each antecedent (A) and consequence (B), the support, confidence, expected confidence, and lift are as in Equations below and fig. 1 (Yusuf, Pratikto and Gerry, 2006). Suppose we define $h \subset\{1$, $2, \ldots \mathrm{P}\}$ and $\mathrm{A}, \mathrm{B} \subset h$ - where $\mathrm{A} \mathrm{U} \mathrm{B}=h$, and $\mathrm{A} \cap \mathrm{B}=\emptyset$. Then, we have that:

$$
P(h)=P(A, B)=\frac{\text { No.of Transactions } A \& B}{\text { No.of Transactions }}
$$

Eq. 1. $\mathrm{P}(\mathrm{h})$ is prevalence or support which yields how often the combination A and B co-occurs.

$$
P(B \mid A)=\frac{P(h)}{P(A)}=\frac{\text { No.of Transactions } A \& B}{\text { No.of Transactions } A}
$$

Eq. 2. $\mathrm{P}(\mathrm{B} \mid \mathrm{A})$ is confidence value which yields the confidence that item $\mathrm{B}$ appears in the basket given $\mathrm{A}$ is already in the basket. Thus, we use the rule $\mathrm{A} \rightarrow \mathrm{B}$.

$$
P(A \mid B)=\frac{P(B)}{P(h)}=\frac{\text { No.of } \text { Transactions } B}{\text { Number of Transactions }}
$$

Eq. 3. $\mathrm{P}(\mathrm{A}) \mathrm{P}(\mathrm{B})$ expected confidence yields the confidence on how frequent items A and items B cooccur in the number of times that the items $\mathrm{B}$ is chosen and placed in the basket.

$$
\text { Lift }=\frac{\text { Confidence }}{\text { Expected Confidence }}
$$

Finally, (4). L (A,B) lift of the rule A $\rightarrow$ B yields a measure of how much more confident we are in item $\mathrm{B}$ given that we see item $\mathrm{A}$ in the basket.

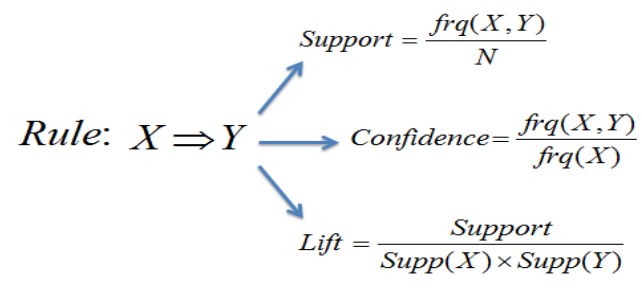

Figure 1. Schematic representation of a rule

MBA is a subset of market research that many researchers are currently paying special attention to with more detailed in [3]. Tang, $\mathrm{K}$, et al [13] proposed an approach to perform market basket analysis in a multistore, multi-period environment. Chen, Y, et al [14] noted that most models used in dealing with market basket problem could not discover any important purchasing patterns when and where multiple stores exist. They developed a method to overcome this weakness; while, Yun, C, et al clustered data of market basket using a novel measurement they named category-based adherence [15]. Cavique, L converted market basket problem into a maximum-weighted clique problem for discovering large item set patterns [6]. According to [16] they developed optimization model for shelf-space management problem in which products are grouped as families and the location of each family is determined on the shelf like cataloging. They considered shelf location effect on sales; but, did not attend the cross-selling effect. Thus, they did not use the purchase data. Nierop, E, et al [17] proposed a method for dealing with shelf-space management problem that consists of two-parts. In the 
first phase, a statistical model was provided to measure the impact of shelf layout on sales. In the second part simulated annealing (SA) was used to maximize expected total profit. They also like [16], did not consider the association rules from customers' purchasing data. Thus, did not use it to maximize cross-selling effect.

Recent researches consider other problems. According to Saraf, R. and Patil, S they proposed a bottomup hierarchical cluster-model for clustering retail items [18]. To do this, they applied the concept of 'distance' between the entities or, groups of entities to achieve the purpose of market-basket analysis. Market basket analysis is now employed by many researchers to other applicable tasks. Shiokawa, Y, et al applied market basket analysis framework to visualize transaction data to assess the various human lifestyles [19]. Solnet, D, et al also studied potentials to grow hotel revenue by exploring most attractive services and products that can attract/satisfy guests and encourage them to repeat their purchase [20]. In furtherance, [5] explore the cultural behaviour of consumers. Further studies can refer to [21-26]. The reviewing of related researches reveal that a main focus of market basket analysis and its application - is geared towards creating a more efficient optimization algorithm for data mining. We can apply evolutionary model and association rule mining [27-32].

\section{MEMETIC BAYESIAN NETWORK EXPERIMENTAL FRAMEWORK}

Evolutionary algorithm seeks to exploit historic numeric data and explore human knowledge via mathematic models and symbolic reasoning to yield an output that is tolerant to imprecision, noise, uncertainty and partial truth as applied to its input [33]. It evolves into meta-rules for constraint satisfaction tasks that use intelligent agents in vector space to seek for optimality. These algorithms/models are inspired by evolution, behavioral patterns in biological populations and nature laws to mimic agents seeking survival [34, 35] as they have proven efficient in complex optimization. Simply put, evolutionary model attempts to explore dynamic processes through exploitation of observed data to yield an output that exhibits robustness, continuous adaptation and flexibility - while displaying the underlying probabilities of data feats of interest. Thus, it seeks an output feat with uncontrollable constraints modeled within the models input that may not be explicitly present in the search space but confined to real parameters as well as limited by boundary values [36-38].

\subsection{Artificial neural network (ANN)}

ANN data processing model is inspired by neurons in the human brain. Thus, consists of interconnected neurons (nodes) with capability to learn by example that makes them universal estimators. As it processes data, its nodes shares data signals and adjust its weights and bias adjustments representing the synapse axons and dendrites to indicate connection strength between synapses respectively [38-39]. Signals are converted so that weights are adjusted as learning occurs and is summed by an adder. Depending on task, its activation function limits its output [40-41] to modulate associated inputs and nonlinear feats exhibited via transfer or activation function as in (5) below:

$$
\emptyset=f(n e t)=f \sum_{i=1}^{m} X i * W i j
$$

ANN attempts to translate into mathematical model, principles of biological processing so as to generate in the fastest time, implicit predictive outcomes of a task [42-43]. Its outcomes are derived from experience, and it is able to recognize feats and behaviours of interest from historic dataset - to yield an optimal solution of high quality and void of over-fitting, irrespective of modification via other approximations with multiple agents. These also, constantly affects the quality of any solution [44]. Its configuration depends on the area to be applied, captured data feats and system requirement. Its connections are set as either explicit (apriori knowledge) and/or implicit (post-priori knowledge) to allow learning so that the net is trained to learn patterns that change its weight and bias based on a rule [40]. Its learning is grouped into either of: supervised, unsupervised and reinforcement $[45,46]$.

The nature of market data is chaotic and requires previous knowledge. Thus, we adopt the recurrent (Jordan) network so that it incorporates previous dataset and previous output to be feedback as input into the model's hidden units, as input into model $[47,48]$ to yield next output. Its correlated weights $\left(\mathrm{W}_{i . j}\right)$ between the input and hidden layers, bias $\left(\mathrm{W}_{o} \mathrm{j}\right)$ and the market basket analysis dataset $\left(\mathrm{x}_{i}\right)$ is summed via the tangent/sigmoid transfer function to yield its output as in (6) and (7) [49].

$$
\begin{aligned}
& Z_{i j}=w_{o} j+\sum_{i=1}^{m} x_{i} * w_{i} j \\
& F\left(Z_{i j}\right)=\frac{2}{1+e^{-2 * Z i j}}-1
\end{aligned}
$$


We construct our Jordan net by modifying the multilayered feedforward with addition a context layer to help retain data between observations. With each move, new inputs are fed in and previous contents in hidden layer is passed into context layer, and later fed back into the hidden layer in the next time-step. The context layer at start is initialized to zero - so that output from the hidden layer on the first iteration will be same as if there is no context layer [50].

The net resolves structural dependencies imposed on it by dataset and hybrid heuristics used via its ability to store earlier data as generated from previous layer(s) [51]. Feed-forward nets are expanded and extended to represent complex dynamic patterns (as our data is rippled with new and previous sets). Feedforward nets treat all data as new so that previous dataset cannot help the model identify data feats, even if such datasets exhibits temporal dependence; causing practical difficulty as network becomes larger. However, Jordan network overcomes this difficulty through its internal feedbacks - making it appropriately suitable for dynamic, non-linear and complex tasks. Thus, output is fed back as input into hidden layer with a time delay [52-53].

Our rationale for the Jordan's network is because it is more plausible and computationally more powerful than others due to use of backpropagation-in-time learning so that its output at time $t$ is used along with new input data to compute its output at time $t+l$ in response to model's dynamic and non-linear feats [51]. Output is computed via Tansig function $\mathrm{y}^{\mathrm{k}}$, which sums input, receives target value of training pattern, computes error data as well as updates weight $c_{j}{ }^{k}$ and bias $c_{o}{ }^{k}$. Error is sent back in next move to input nodes from output via error-backpropagation to correct the weights and find those that approximates to the target output with selected accuracy. Weights are modified by minimizing error between target and computed outputs as forward pass ends. If the error is higher than selected value, process continues with reverse pass; else, training stops [54-55].

Its training aim at best fit weight dataset that assumes approximation influence of data points at the center - so that function decreases with distance from its center. Its Euclidean length $\left(\mathrm{r}_{\mathrm{j}}\right)$ yields distance between datum vector $\mathrm{y}=\left(\mathrm{y}_{1}, \ldots, \mathrm{y}_{\mathrm{m}}\right)$ and center $\left(\mathrm{w}_{1 j}, \ldots, \mathrm{w}_{m j}\right)$ as in $(8)[48,49]$ :

$$
r_{j}=|| y-Y^{j} \|=\left\{\sum_{i=1}^{m}\left(y_{i}-w_{i} j\right)^{2}\right\}^{1 / 2}
$$

The suitable transfer function is applied to $r_{j}$ to yield (9):

$$
\emptyset\left(r_{j}\right)=\emptyset \| y-Y^{j}||
$$

Finally, output k receives weighted combination as in (10):

$$
y^{k}=w_{o}+\sum_{j=1}^{n}\left(c_{j}^{k} * \emptyset\left(r_{j}\right)\right)=w_{o}+\sum_{j=1}^{n}\left(c_{j}^{k} * \emptyset\left\|y-Y^{j}\right\|\right)
$$

\subsection{Genetic algorithm (GA)}

GA is inspired by Darwinian genetic evolution (survival of fittest) consists of population (data) chosen for selection with potential solutions to a specific task. Each potential solution is an individual for which optimal is found using four operators: initialize, select, crossover and mutation $[33,56]$. Individuals with genes close to optimal, is said to be fit. Fitness function determines how close an individual is to optimal solution. Ojugo, A.A, et al [38] notes the operators as:

a. Initialize - Individual data are encoded into forms suitable for selection. Each encodings type used has its merit. Binary encodings are computationally more expensive. Decimal encoding has greater diversity in chromosome and greater variance of pools generated; float-point encoding or its combination is more efficient than binary. Thus, it encodes as fixed length vectors for one or more pools of different types. The fitness function evaluates how close a solution is to its optimal - after which they are chosen for reproduction. If solution is found, function is good; else, is bad and not selected for crossover. The fitness function is the only part with knowledge of task. If more solutions are found, the higher its fitness value.

b. Selection - best fit individuals close to optimal are chosen to mate. The larger the number of selected, the better the chances of yielding fitter individuals. This continues until one is chosen, from the last two/three remaining solutions, to become selected parents to new offspring. Selection ensures the fittest individuals are chosen for mating but also allows for less fit individuals from the pool and the fittest to be selected. A selection that only mates the fittest is elitist and often leads to converging at local optima.

c. Crossover ensures best fit individual genes are exchanged to yield a new, fitter pool. There are two crossover types (depends on encoding type used): (a) simple crossover for binary encoded pool. It allows single- or multi-point cross with all genes from a parent, and (b) arithmetic crossover allows new pool to be created by adding an individual's percentage to another. 
d. Mutation alters chromosomes by changing its genes or its sequence, to ensure new pool converges to global minima (instead of local optima). Algorithm stops if optimal is found, or after number of runs if new pools are created (though computationally expensive), or when no better solution is found. Genes may change based on probability of mutation rate. Mutation improves the much needed diversity in reproduction and its algorithm is as thus:

Cultural GA is a variants of GA with a belief space define as thus: (a) Normative (has specific value ranges to which an individual is bound), (b) Domain (has data about task domain), (c) Temporal (has data about events' space is available), and (d) Spatial (has topographical data). In addition, an influence function mediates between belief space and the pool - to ensure and alter individuals in the pool to conform to belief space. CGA is chosen to yield a pool that does not violate its belief space and helps reduce number of possible individuals GA generates till an optimum is found [56, 34, 37, 38].

\section{MATERIALS AND METHODOLOGY}

\subsection{Problem description and formulation}

Consider market data logs that include items purchased by customers. The manager of a supermarket wants to maximize the interestingness of the product placement on shelves. The interestingness value is related to mined association rules and each item's location on shelves [57]. The rationale for the interestingness maximization with location considerations is based on the fact that, association rule mining helps maximize cross-selling effect of items [58]. It is also clear that the location of shelves has the undeniable impact on the selling rate of items. Thus, items that are placed near the entrance or exit doors have more chance to be purchased. So, preference function of the store's manager depends on the following parameters: selling benefit, support and confidence of each pair of items, and the selling possibility of each item from each shelf [59]. These parameters are thus integrated into the preference function (pf) as in (11):

$$
p f=\sum_{i=1}^{m-1}\left[\sum_{l=i+1}^{m}\left[C_{i l}+C_{l i} \sum_{k=1}^{p}\left[b_{i} v_{i k}+b_{i} v_{i k} x_{i k} x_{l k}\right]\right]\right]
$$

$m$ is the number of items, $p$ is the number of shelves, $\mathrm{C}_{i l}$ is the confidence of the rule (item $i \rightarrow$ item $l$ ), $\mathrm{b}_{i}$ is selling benefit of the $i$ th item, $\mathrm{v}_{i k}$ is the selling possibility degree of the item $i$ if and when placed into the $k$ th-shelf, and $\mathrm{x}_{i k}$ is binary decision variable that takes value of 1 when the item $i$ is allocated to the shelf $k$; Otherwise, its value is 0 . There are restrictions that limit preference function value. Thus, capacity limitation $(c l)$ of each shelf must be considered as the following constraint:

$$
c l=\sum_{i=1}^{m} x_{i k} \leq U_{k} \text { with } k=1,2, \ldots P
$$

$\mathrm{U}_{\mathrm{k}}$ is the capacity of the $k$ th-shelf. The second constraint is the association constraint such that support of the rule (item $i \rightarrow$ item $l$ ) must be greater than minimum threshold determined by the decision maker. The objective function and constraints are non-linear functions in which decision variables are binary. Thus, we will deal with a rough feasible space that increases the probability of trapping in the local optimum. Thus, our need for the use of an evolutionary unsupervised model in the scenario thus presented.

\subsection{Numeric example dataset}

Dataset is retrieved from Delta Mall (Shoprite) Asaba and Warri respectively dataset as in Table 1 below. Table 1 shows the encoded market basket dataset of items as they are co-selected of the various shelves and placed in the basket at the same time. For example, S01 for Item 1 has a prevalence of 0.81 . This implies that there is $81 \%$ chance that items 1, 2, 6 and 8 are picked from shelf S01 and placed in the basket. The Delta Mall market basket dataset was employed to simulate the model as well as yield cum describe the proposed model-based solution. Thus, the system shows eight items that must be allocated into four-shelves. Also, based on the shelf's positions, each shelf has a different impact on the selling possibility of allocated goods, and these possibilities were determined by economists and experts as presented in Table 1 .

Table 1 . Encoded binary representation of 5-baskets/shelve values for analysis

\begin{tabular}{ccccccccc}
\hline $\begin{array}{c}\text { Items } \\
\text { Shelves }\end{array}$ & 1 & 2 & 3 & 4 & 5 & 6 & 7 & 8 \\
\hline S01 & 1 & 1 & 0 & 0 & 0 & 1 & 0 & 1 \\
S02 & 0 & 1 & 0 & 1 & 1 & 0 & 1 & 0 \\
S03 & 0 & 0 & 1 & 0 & 1 & 0 & 1 & 1 \\
S04 & 1 & 0 & 1 & 1 & 1 & 0 & 0 & 0 \\
\hline (Source: Authors' own processing and translation)
\end{tabular}




\subsection{Model design problems}

Issues to be resolved in the model design include:

a. Many studies aim at single heuristic to globally classify data or rules into various classes. This has often yielded false-positives (classifying a rule as genuine when it is false) and true-negatives (inability of model to classify a rule) error results.

b. Such models employ hill climbing methods that often gets their solution trapped at local minima because their speed shrinks as such models often approaches its optima.

c. Resolving conflict issues in structured learning and from statistical dependencies imposed by data and the use of multiple methods adopted/adapted, is quite a tedious.

\subsection{Model design goals and objective}

The proposed system aims to solve the existing problem of market basket analysis utilizing the following properties, which is in tandem to [60-62]:

a. Embody the knowledge of human experts with the help of special software tools, manipulate data to solve problems and make decisions in that domain.

b. Processes are better formalized and defined on machines.

c. Knowledgebase update is automatic

d. Processes are better formalized and defined on machines.

\subsection{Experimental model / algorithm framework}

The proposed model consists of four parts:

a. Knowledgebase consists of historic, observed-structured items co-occurrence dataset (feats) of market basket for Delta Mall. These have been gracefully encoded as if-then rules - hereby represented as optimized binary functions for the selected data feats.

b. Inference engine consists of hybrid associative rules and the genetic algorithm trained neural network model. Thus, the inference engine seeks to infer consequents derived from antecedents that have been trained using the hybrid memetic algorithm. The rules represent selected data feats of interest encoded as if-then (rule-based) conditions with possible outcomes and actions (classified into support, confidence and lift classes) upon criterion score being met or achieved. The Jordan network provides a self-learning machine, better tuned for robustness via genetic algorithm optimizer that yields greater flexibility of the rule-based data. Thus, it adapts the system to autonomously classify the market basket data into varying class-types as well as yield centralized-scaled boundary in determining high or low degree membership function.

c. Decision support - consists of the predicted output and the output database that is updated automatically in time as patients are diagnoses as long as it encounters and read sin new data. The decision support predicts system output based on the cognitive and the emotional filers as display by the output device. This is seen in Figure 2.

Model is first initialized with rules. Individual solutions are selected from pool via tournament method to determine the candidates to mate and yield next generation. Crossover and mutation is applied to help network learn dynamic and non-linear feats in the dataset and feats of interest using a multi-point crossover. With mutation, data are randomly generated using Gaussian distribution corresponding to crossover points (all genes are from single parent). As new parents contributes to yield new pool, mutation is applied to yield random genes from which three-candidates are selected (and allocated new random values that conforms to boundary limits) to undergo further mutation. The number of mutation applied depends on how far GA is progressed on the network (how fit is the fittest individual in the pool), which equals fitness of the fittest individual divided by 2 . New individuals replace old with low fitness so as to create a new pool. Process continues until individual with a fitness of 0 is found. Thus, solution has been reached. Initialization/Selection with ANN ensures the first 3-beliefs are met; while, mutation ensures fourth-belief is met. Its influence function determines the number of mutations that take place, and knowledge of solution (i.e. how close solution is) has direct impact on how model is processed. The GANN model pseudocode is as thus:

INPUT:

1. Pool size (k), crossover (c), mutation (v), influence functn (Ifnc) and n; // Initialization and Selection

2. Randomly generate $K$ possible solution

3. Save solution in population Kok; // Loop till terminal point

4. For $\mathrm{m}=1$ to $\mathrm{n}$ do; // Crossover

5. Number of crossover $\mathrm{nc}=(\mathrm{k}-$ Ifnc $) / 2$;

6. For $\mathrm{u}=1$ to $\mathrm{n}$ do;

7. Select two solutions randomly $\mathrm{E}_{\mathrm{A}}$ and $\mathrm{F}_{\mathrm{G}}$ for $K$; 
8. Generate $\mathrm{G}_{\mathrm{V}}$ and $\mathrm{H}_{\mathrm{N}}$ by 2-point crossover to $\mathrm{E}_{\mathrm{A}}$ and $\mathrm{F}_{\mathrm{G}}$;

9. Save $\mathrm{G}_{\mathrm{V}}$ and $\mathrm{H}_{\mathrm{N}}$ to $K 2$;

10. End For; //Mutation

11. For $\mathrm{u}=1$ to $\mathrm{n}$ do;

12. Selection a solution $\mathrm{Y}_{\mathrm{h}}$ from $K 2$;

13. Mutate each bit of $Y_{h}$ under Ifnc

14. Generate a new solution $\mathrm{Y}_{\mathrm{h}}{ }^{\mathrm{i}}$

15. If $\mathrm{Y}_{\mathrm{h}}{ }^{\mathrm{i}}$ is impossible

16. Recompute $\mathrm{Y}_{\mathrm{h}}{ }^{\mathrm{i}}$ with possible solution by modifying $\mathrm{Y}_{\mathrm{h}}{ }^{\mathrm{i}}$

17. End if

18. Recompute $\mathrm{Y}_{\mathrm{h}}$ with $\mathrm{Y}_{\mathrm{h}}{ }^{\mathrm{i}}$ in $K 2$

19. End for //Recompute

20. Recompute $K=K 2$;

21. Return Best solution in Y

Model stops if stop criterion zero (0) is met; Or, if number of epochs is reached. Thus, number of sets of size $k$ picked from $\mathrm{p}$ items yields:

$$
\left(\begin{array}{l}
p \\
k
\end{array}\right)=\frac{p !}{k !(p-k) !} \equiv \frac{8 !}{4 !(8-4) !}=105 \text { item combination }
$$

\subsection{Result findings and discussion}

The result simulated association of basket analysis dataset is shown in Table 2. Another feature of major concern and impact to the market basket data in the allocation of items to shelves is the selling benefit. So, it is logical that in maximizing expected benefit of the selling, the products with the higher benefits must be allocated to shelves with higher selling possibilities. Table 3 shows the values of the products' benefit. Table 4 and Table 5 show the support and confidence simulated values respectively.

Table 2. Simulated association of basket analysis dataset

\begin{tabular}{ccccccccc}
\hline $\begin{array}{c}\text { Items } \\
\text { Shelves }\end{array}$ & 1 & 2 & 3 & 4 & 5 & 6 & 7 & 8 \\
\hline S01 & 0.81 & 0.43 & 0.12 & 0.90 & 0.22 & 0.25 & 0.34 & 0.87 \\
S02 & 0.51 & 0.21 & 0.55 & 0.19 & 0.90 & 0.71 & 0.12 & 0.21 \\
S03 & 0.32 & 0.82 & 0.61 & 0.28 & 0.21 & 0.23 & 0.19 & 0.12 \\
S04 & 0.54 & 0.76 & 0.11 & 0.58 & 0.31 & 0.46 & 0.01 & 0.40 \\
\hline
\end{tabular}

(Source: Authors' own processing and translation)

Table 3. Selling possibility of each item to purchased $\left(\mathrm{V}_{i k}: i=1,2, \ldots 8 ; \mathrm{k}=1,2,3,4\right)$

\begin{tabular}{ccccccccc}
\hline $\begin{array}{c}\text { Items } \\
\text { Shelves }\end{array}$ & 1 & 2 & 3 & 4 & 5 & 6 & 7 & 8 \\
\hline S01 & 0.81 & 0.43 & 0.12 & 0.90 & 0.22 & 0.25 & 0.34 & 0.87 \\
S02 & 0.51 & 0.21 & 0.55 & 0.19 & 0.90 & 0.71 & 0.12 & 0.21 \\
S03 & 0.32 & 0.82 & 0.61 & 0.28 & 0.21 & 0.23 & 0.19 & 0.12 \\
S04 & 0.54 & 0.76 & 0.11 & 0.58 & 0.31 & 0.46 & 0.01 & 0.40 \\
\hline
\end{tabular}

Table 4. Support values of simulated data $\left(\mathrm{S}_{i l}\right)$

\begin{tabular}{ccccccccc}
\hline $\begin{array}{c}\text { Items } \\
\text { Shelves }\end{array}$ & 1 & 2 & 3 & 4 & 5 & 6 & 7 & 8 \\
\hline 01 & 0.42 & 0.31 & 0.12 & 0.09 & 0.14 & 0.28 & 0.13 & 0.42 \\
02 & 0.00 & 0.39 & 0.18 & 0.11 & 0.19 & 0.34 & 0.19 & 0.29 \\
03 & 0.00 & 0.00 & 0.46 & 0.19 & 0.22 & 0.11 & 0.35 & 0.32 \\
04 & 0.00 & 0.00 & 0.00 & 0.41 & 0.18 & 0.07 & 0.19 & 0.21 \\
05 & 0.00 & 0.00 & 0.00 & 0.00 & 0.36 & 0.02 & 0.21 & 0.01 \\
06 & 0.00 & 0.00 & 0.00 & 0.00 & 0.00 & 0.49 & 0.21 & 0.12 \\
07 & 0.00 & 0.00 & 0.00 & 0.00 & 0.00 & 0.00 & 0.44 & 0.32 \\
08 & 0.00 & 0.00 & 0.00 & 0.00 & 0.00 & 0.00 & 0.00 & 0.32 \\
\hline (Source: Author's own processing) & & & & & &
\end{tabular}


Table 5. Confidence values of simulated data $\left(\mathrm{C}_{i l}\right)$

\begin{tabular}{ccccccccc}
\hline $\begin{array}{c}\text { Items } \\
\text { Shelves }\end{array}$ & 1 & 2 & 3 & 4 & 5 & 6 & 7 & 8 \\
\hline 01 & 1 & 0.52 & 0.43 & 0.24 & 0.35 & 0.49 & 0.13 & 0.10 \\
02 & 0.52 & 1 & 0.35 & 0.25 & 0.63 & 0.03 & 0.21 & 0.30 \\
03 & 0.43 & 0.33 & 1 & 0.20 & 0.38 & 0.39 & 0.13 & 0.21 \\
04 & 0.23 & 0.24 & 0.20 & 1 & 0.67 & 0.32 & 0.13 & 0.12 \\
05 & 0.36 & 0.65 & 0.39 & 0.67 & 1 & 0.88 & 0.31 & 0.26 \\
06 & 0.49 & 0.01 & 0.41 & 0.32 & 0.87 & 1 & 0.11 & 0.16 \\
07 & 0.11 & 0.19 & 0.10 & 0.12 & 0.29 & 0.10 & 1 & 0.23 \\
08 & 0.09 & 0.32 & 0.19 & 0.11 & 0.26 & 0.16 & 0.24 & 1 \\
\hline (Source: Author's
\end{tabular}

(Source: Author's own processing)

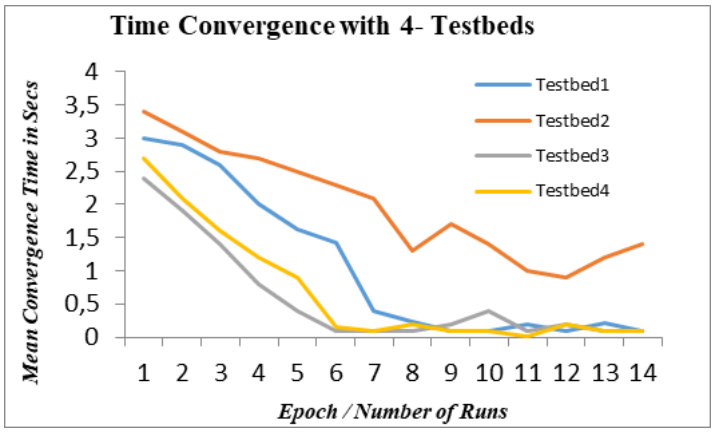

Figure 2. Evolution Convergence Time Using 4-Testbeds

Lastly, Figure 2 shows execution time versus convergence using four (4) separate test-beds to simulate the effectiveness and efficiency of the model. With data logs of items purchased by customers, the proposed model-based solution converges faster as items commonly placed in a basket are selected [63-64]; and thus, yields an effective means to maximize the interestingness of product placement on the shelves. This interestingness value(s) are rules mined by association using the frequency growth path-algorithm for item location on the shelves [65-69]. The rationale for interestingness maximization with location considerations is based on the fact that, association rule mining helps to maximize cross-selling effect of items. Also, it is clear that the location of shelves has the undeniable impact on the selling rate of items. Thus, items that are placed near the entrance or exit doors have more chance to be purchased. So, preference function of the store's manager depends on the following parameters: selling benefit, support and confidence of each pair of items, and the selling possibility of each item from each shelf.

\section{CONCLUSION}

Our memetic (genetic algorithm trained neural net) model as used for classification of market basket data - adapts GA to help speed up the final stages of ANN and thus, yield a robust optima in the shortest amount of time for such a dynamic and complex task. The rule-based heuristics will help better represent data values in the model [70-72]. Though, hybrids are quite difficult to implement, exploit and explore - it however yields better solutions with appropriate parameter selection that must be encoded through the model's structured learning. This will in turn help address the issues of statistical dependencies imposed on hybrid by the underlying stochastic heuristics adopted, resolve conflicts imposed with encoding of dataset cum data feats of interest [73] as well as highlight the implications of such multi-agent model as agents seek to create their own behavioural rules on the dataset used - as the model proposes a solution to display underlying probabilities of data feats of interest. GA helps to yield better generation via its process of recombination and mutation as applied.

\section{REFERENCES}

[1] Aguinis, H, Forcum, L.E, and Joo H. "Using market basket analysis in management research," Journal of Management, vol. 39(7), pp. 1799-1824, 2013.

[2] Prithiviraj, P and Porkodi, R. "A comparative analysis of association rule mining algorithms in data mining," American J. of Computer Science and Engineering Survey, vol. 3(1), pp. 98-119, 2015. 
[3] Barbu, A. "Eight contemporary trends in the market research industry," Management and Marketing, vol. 8(3), pp. 429-450, 2013.

[4] Ojugo, A.A and Chiemeke, S. "Inventory management using the market basket analysis: a comparative review of fpgrowth and apriori algorithm," Journal of Computer Science Application - Nigerian Computer Society, vol. 56(4), pp. 21-34, 2019.

[5] Coscia, C, Fontana, R, and Semeraro, P. "Market Basket Analysis for studying cultural Consumer Behaviour: AMTP Card-Holders," Statistica Applicata, vol. 26(2), pp73-92, 2016.

[6] Cavique, L. "A scalable algorithm for the market basket analysis," Journal of Retailing and Consumer Services, vol. 14(6), pp. 400-407, 2007.

[7] Kaur, M and Kang, S. "Market Basket Analysis: Identify the Changing Trends of Market Data Using Association Rule Mining," International Conference on Computational Modelling and Security (CMS 2016), vol. 85, pp78-85, 2016.

[8] Heydary, $\mathrm{M}$ and Yousefli, A. "A new optimization model for market basket analysis with allocation considerations: A genetic algorithm solution approach," Management \& Marketing. Challenges for the Knowledge Society, vol. 12(1), pp. 1-11, 2017.

[9] Ojugo, A.A. "Unsupervised cluster prediction model for market basket analysis: quest for better alternatives to association rule mining approach," In Proc. of the 21st ISTEAMS Conf. on Intelligent Systems and Management of Data, pp. 121-135, Retrieved Sep 242018.

[10] Ojugo, A.A, Yoro, R.E, and Okobah, I.P. "A comparative study of market basket analysis rule mining approaches," Technical Report of the Federal University of Petroleum Resources Effurun (FUPRE-TRON-1023), pp. 312-337, 2018.

[11] Dhaenens, C and Jourdan, L. "Metaheuristics and Association Rules, in Dhaenes, C. and Jourdan, L. (Eds.), Metaheuristics for Big Data," John Wiley \& Sons, Hoboken, pp. 87-108, 2016.

[12] Ojugo, A.A and Yoro, R.E. "Intelligent lightweight market basket associative rule mining for smartphone cloudbased application to ease banking transactions," In Proc. of the 21st ISTEAMS Conf. on Intelligent Systems and Management of Data, pp. 23-37, 2018.

[13] Tang, K, Chen, Y and Hu, H. "Context-based market basket analysis in a multiple-store environment," Decision Support Systems, vol. 45(1), pp. 150-163, 2008.

[14] Chen, Y, Tang, K, Shen, R, and Hu, Y. "Market basket analysis in a multiple store environment," Decision Support Systems, 40, pp. 339-354, 2005.

[15] Yun, C, Chuang, K, and Chen, M. "Adherence clustering: an efficient method for mining market-basket clusters," Information Systems, 31, pp. 170-186, 2006.

[16] Russell, R.A and Urban, T.L. "The location and allocation of products and product families on retail shelves," Annuals of Operation Research, vol. 179(1), pp. 131-147, 2010.

[17] Nierop, E, Fok, D and Franses, P. "Interaction between shelf layout and marketing effectiveness and its impact on optimizing shelf arrangements," Marketing Science, vol. 27(6), pp. 1065-1082, 2006.

[18] Saraf, R and Patil, S. "Market-Basket Analysis using Agglomerative Hierarchical approach for clustering a retail items," International Journal of Computer Science and Network Security, vol. 16(3), pp. 47-56, 2016.

[19] Shiokawa, Y, Misawa, T, Date, Y, and Kikuchi, J. "Application of market basket analysis for the visualization of transaction data based on human lifestyle and spectroscopic measurements," Analytical chemistry, vol. 88(5), pp. 2714-2719, 2016.

[20] Solnet, D, Boztug, Y and Dolnicar, S. "An untapped gold mine? Exploring the potential of market basket analysis to grow hotel revenue," International Journal of Hospitality Management, 56, pp. 119-125, 2016.

[21] Holý, V, Sokol, O, and Černý, M. Clustering Retail Products Based on Customer Behavior, Applied Soft Computing, In Press, Accepted Manuscript, 2017.

[22] Shrivastava, S and Rajput, V. "Evolutionary algorithm-based association rule mining: A brief survey," International Journal of Innovation in Engineering research and Management, vol. 2(1), pp. 1-7, 2015.

[23] Meisel, S and Mattfeld, D. "Synergies of Operations Research and Data Mining," European Journal of Operational Research, vol. 206(1), pp. 1-10, 2010.

[24] Sadh, A.S and Shukla, N. "Association rules optimization: A survey," International Journal of Advanced Computer Research, vol. 3(9), pp. 111-115, 2013.

[25] Sharma, A and Tivary, N. "A survey of association rule mining using genetic algorithm," International J. of Computer Applications \& Information Technology, vol. 1(2), pp. 5-11, 2012.

[26] Tiwari, R and Singh, M.P. "Correlation-based Attribute Selection using Genetic Algorithm," International Journal of Computer Application, vol. 4(8), pp. 28-34, 2010.

[27] Tomar, N and Manjhvar, A.K. "A survey on data mining optimization techniques," International Journal of Science Technology \& Engineering, vol. 2(6), pp. 130-133, 2015.

[28] Awodele, O, Izang, A.A, Kuyoro, and S Osisanwo, F. "Big Data and Cloud Computing Issues," International Journal of Computer Applications 0975-8887, vol. 133(12), 2016.

[29] Annie, L and Kumar, A. "Market basket analysis for a Supermaket Based on Frequent item Mining," International Journal of Computer Science, vol. 9(5), pp. 34-43, 2012

[30] Agrawal, R, Imielinski, R, and Swami, H. 'Mining association between sets of items in massive databases," In Proc. of ACM SIGMOD Conference on Management of Data, pp. 207-216, Chicago, Washington DC, USA, 1993.

[31] Agrawal, R and Srikant, R. "Fast algorithms for mining association rules in large databases," Proc. of 20th Int. Conf. on Very Large Databases, pp. 487-499, 1994. 
[32] Vijayata, W and Debajyoti, M. "Mobile Agent based Market Basket Analysis on Cloud," India. 2014 International Conference on IT. (pp. 49-62). Pune, India: Department Of Information Technology, Maharashtra. Institute of Technology Pune, India, 2014.

[33] Coello, C, Pulido, G, and Lechuga, M, "Handling multiple objectives with particle swarm optimization," Proceedings of Evolutionary Computing, vol. 8(3), pp. 256-279, 2004.

[34] Ojugo, A, Eboka, A, Okonta, E, Yoro, R, and Aghware, F. "GA rule-based intrusion detection system," Journal of Computing and Information Systems, vol. 3(8), pp. 1182-1194, 2012.

[35] Ojugo, A.A and Yoro, R. "Computational intelligence in stochastic solution for Toroidal Queen," Progress in Intelligence Computing and Applications, vol. 2(1), pp. 46-56, 2013.

[36] Ojugo, A.A, Emudianughe, J, Yoro, R.E, Okonta, E.O, and Eboka, A. "Hybrid neural network gravitational search algorithm for rainfall runoff modeling," Progress in Intelligence Computing and Application, vol. 2(1), pp. 22-33, 2013.

[37] Ojugo, A.A, Ben-Iwhiwhu, E, Kekeje, D.O, Yerokun, M.O, and Iyawa, I.J.B. "Malware propagation on time varying network," Int. J. Modern Edu. Comp. Sci., vol. 8, pp. 25-33, 2014.

[38] Ojugo, A.A, Eboka, A.O, Yoro, R.E, Yerokun, M.O, and Efozia, F.N. "Hybrid model for early diabetes diagnosis," Mathematics and Computers in Science and Industry (A Mathematics and Computers in Science and Engineering Series), series 50, pp. 176-182, 2015.

[39] Fausett L. Fundamentals of Neural Networks, New Jersey: Prentice Hall, pp. 240, 1994.

[40] Bishop, C. M. Neural networks for pattern recognition, Oxford University Press, ISBN: 0-342-45230, pp. 253-294, 1995.

[41] Guo, W.W and Xue, H. 'An incorporative statistic and neural approach for crop yield modelling and forecasting," Neural Computing and Applications, vol. 21(1), pp. 109-117, 2011.

[42] Hall, M. J. "How well does your model fit data?," Journal of Hydroinformatics, vol. 3(1), pp. 49-55, 2001.

[43] Haykin, S. Neural networks, comprehensive foundation, Prentice Hall: New Jersey, pp. 23-89, 1999.

[44] Heppner, H and Grenander, U. Stochastic non-linear model for coordinated bird flocks, In Krasner, S (Ed.), The ubiquity of chaos, pp. 233-238, Washington: AAAS, 1990.

[45] A.A. Ojugo and A.O. Eboka. "Signature-based malware detection using approximate Boyer Moore string matching algorithm," Int. J. of Mathematical Sciences and Computing, vol. 3(5), pp. 49-62, 2019.

[46] R.E. Yoro and A.A. Ojugo. "An intelligent model to predict the relationship of weather conditions for fish farming production yield in Nigeria," American Journal of Modeling and Optimization, vol. 4(3), pp. 79-90, 2019.

[47] R.E. Yoro and A.A. Ojugo. "Quest for prevalence rate of Hepatitis-B Virus infection in Nigeria: comparative study of supervised versus unsupervised model," American J. of Modeling and Optimization, vol. 4(3), pp. 79-90, 2019.

[48] A.A. Ojugo and A.O. Eboka. "Modeling the computational solution of market basket associative rule mining approaches using deep neural network," Digital Technologies, vol. 3(1), pp. 1-8, 2018.

[49] A.A. Ojugo and A.O. Eboka. "Comparative evaluation for performance adaptive model for spam phishing detection," Digital Technologies, vol. 3(1), pp. 9-15, 2018.

[50] Perez, M and Marwala, T. "Stochastic optimization approaches for solving Sudoku," IEEE Transaction on Evol. Comp., pp. 256-279, 2011.

[51] Mandic, D and Chambers, J. Recurrent Neural Networks for Prediction: Learning Algorithms, Architectures and Stability, Wiley \& Sons: New York, pp. 56-90, 2001.

[52] Inan, G and Elif, D. Adaptive neuro-fuzzy inference system for classification of EEG using wavelet coefficient 2005). Retrieved from: http://www.rorylewis.com/PDF/04

[53] Ludmila I. K. "Fuzzy classifiers," Scholarpedia, 3(1), pp 2925, 2008.

[54] Ursem, R, Krink, T, Jensen, M, and Michalewicz, Z. "Analysis and modeling of controls in dynamic systems," IEEE Transaction on Memetic Systems and Evolutionary Computing, vol. 6(4), pp. 378-389, 2002.

[55] Minns, A. Artificial neural networks as sub-symbolic process descriptors, published PhD Thesis, Balkema, Rotterdam, Netherlands, 1998.

[56] Reynolds, R. "Introduction to cultural algorithms," Transaction on Evolutionary Programming (IEEE), pp. 131-139, 1994.

[57] Olson, D.L. "Market Basket Analysis, Chapter Descriptive Data Mining," Part of the series: Computational Risk Management, pp. 29-41. 2016.

[58] Waiswa, P.P.W and Baryamureeba, V., "Extraction of interesting association rules using Genetic Algorithms," International Journal of Computing and ICT Research, vol. 2(1), pp. 26-33, 2008.

[59] Woo, J. "Market Basket Analysis using Spark," ARPN Journal of Science and Technology, vol. vol.4, pp. 207-209, 2015.

[60] Marafi, S. Market Basket Analysis with R, 2014. Retrieved from: http://www.salemmarafi.com/code/market-basketanalysis-with-r/.

[61] Rosaria, S. (2015). How to build a Market Basket Analysis Engine. Retrieved from Analyticbridge: http://www.analyticbridge.datasciencecentral.com/profiles/blogs/how-to-build-a-market-basket-analysis-engine.

[62] Maheshwari, N, Pandey, N. K, and Agarwal, P. "Market Basket Analysis using Association Rule Learning," International Journal of Computer Applications, 0975-8887, 2016.

[63] Komal Khurana and Mrs. Simple Sharma. "A Comparative Analysis of Association Rules Mining Algorithms," International Journal of Scientific and Research Publications, vol. 3(5), May 2013.

[64] Agrawal, R, Imielinski, R, and Swami, S. "A Mining association Between Sets of Items in Massive Databases," In Proc. of ACM SIGMOD Conference on Management of Data, pp. 207-216, 1993. Retrieved September 24, 2016 
[65] Gagandeep, K and Aggarwal, S. "Performance Analysis of Association Rule Mining Algorithms," International Journal of Advanced Research in Computer Science and Software Engineering, vol. 3(8), 2013.

[66] Parita Parikh and Dinesh Waghela. "Comparative Study of Association Rule Mining Algorithms, Parita Parikh et al," UNIASCIT, vol. 2(1), pp. 170-172, 2012.

[67] Borgelt, C. (2014). Find frequent itemsets and association rules with the Apriori Algorithm. Retrieved from: C. Borgelt's home page: http://www.borgelt.net/doc/apriori/apriori.html.

[68] Girotra, M, Nagpal, K, Inocha, S, and Sharma, N. "Comparative Survey on Association Rule Mining Algorithms," International Journal of Computer Applications, vol 84(10), 2013.

[69] Shendge, P and Gupta, T. "Comparitive Study of Apriori \& FP Growth Algorithms," Indian journal of research, vol. 2(3), Mar 2013.

[70] Dhanabhakyam, M and Punithavalli, M. "A Survey on Data Mining Algorithm for Market Basket Analysis," Global Journal of Computer Science and Technology, vol. 11(11), pp. 13-21, 2011.

[71] Ming-Syan, C, Han, J, and Yu, P.S. "Data mining: an overview from a database perspective," IEEE Transactions on Knowledge and Data Engineering, vol. 8(6), 2016.

[72] Meherwar, F and Maruf, P. 'Survey of Machine Learning Algorithms for Disease Diagnostic," Journal of Intelligent Learning Systems and Applications, pp. 1-16, 2017.

[73] Kumbhare, T.A and Chobe, S. "An overview of association rule mining algorithms," International Journal of Computer Science and Information Technology, vol. 5(1), pp. 927-930, 2014.

\section{BIOGRAPHIES OF AUTHORS}

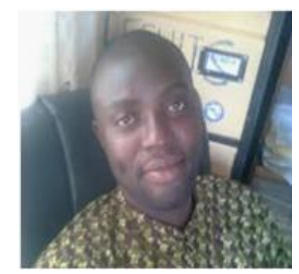

Arnold Adimabua Ojugo received BSc in Computer Science from the Imo State University Owerri in 2000, MSc, also in Computer Science from the Nnamdi Azikiwe University Awka in 2005, and $\mathrm{PhD}$ in Computer Science from Ebonyi State University

Abakiliki in 2013. He is currently, a Reader/Associate Professor with the Department of Mathematics/Computer Science of the Federal University of Petroleum Resources Effurun, Delta State, Nigeria. His research interests: Intelligent Systems and Control, Machine-Learning, Performance Computing, Computer Vision, Ubiquitous Computing, Data Security and Graph Theory. He is an Editor with the Progress for Intelligent Computation and Application, Advancement for Scientific and Engineering Research and SciencePG (ACIS, AJNC, NET and WCMC Journals). He is also a member of: The Nigerian Computer Society, Computer Professionals of Nigeria and International Association of Engineers (IAENG).

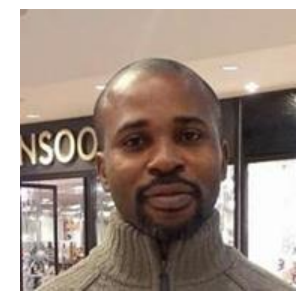

Andrew O. Eboka `received his HND in Computer Science from Akanu Ibiam Federal Polytechnic in the year 1998, Ebonyi State, PGD from Ebonyi State University in 2013, BSc/Ed in Computer Science Education from the Enugu State University of Science and Technology, Enugu in 2013. $\mathrm{He}$ received his MSc in Network Computing from Coventry University, United Kingdom. He currently lectures with the Department of Computer Science Education at Federal College of Education (Technical), Asaba, Delta State, Nigeria. His research interests include: Network Security and Management, CyberSecurity, Ubiquitous Computing, Data Security and Forensics. $\mathrm{He}$ is a member of: The British Computer Society (BCS), Association of Computer Machinery (ACM). Computer Professionals of Nigeria (MCPN) and The Nigerian Computer Society, Computer Professionals of Nigeria and International Association of Engineers (IAENG). 